Results: the IGRA-positive rates were $20.6 \%$ (206/1000). Forty-eight patients with a IGRA-positive finished the treatment with rifampin for 4 months. 2-years follow-up shows no latent tuberculosis developed into active tuberculosis in the patients with prevention of rifampin, while $3(0.38 \%)$ patients with negative IGRA and $22(10.7 \%)$ patients with positive IGRA without rifampin treatment developed into active tuberculosis. 25 patients had active tuberculosis disease in two years, which 24 patients with SLE and one patient with systemic vasculitis while 20 cases had pulmonary tuberculosis, 3 cases had vertebral tuberculosis and tuberculous peritonitis, tuberculous meningitis was 1 case, respectively. Univariate analysis showed that age, entities of rheumatic disease, dosage of glucocorticoid, DMARDs using, comorbidity with interstitial lung disease and cancer were significantly associated with tuberculosis activation $(P<0.05)$, with the ORs of $0.959,0.592,4.45,0.226, A 3.51$ and 69.9 , respectively. Entities of rheumatic diseases, Dosage of glucocorticoid, DMARDs using, comorbidity of cancer entered the final multivariate Logistic model. No severe adverse effects occurred in all subjects.

Conclusions: Medium or high dosage of glucocorticoid treatment appears to increase the risk of activation of latent tuberculosis infection. Latent tuberculosis activation could be safely prevented by 4-months rifampin treatment while starting glucocorticoid and DMARDs therapy.

Disclosure of Interest: None declared

DOI: 10.1136/annrheumdis-2017-eular.3937

\section{SAT0708 SYMPTOMS INDICATIVE OF INFLAMMATORY ARTHRITIS ARE COMMON IN THE PRIMARY CARE POPULATION: FINDINGS FROM THE SYMPTOMS IN PERSONS AT RISK OF RHEUMATOID ARTHRITIS SURVEY}

S.L. Hider ${ }^{1,2}$, S. Muller ${ }^{1}$, J. Prior ${ }^{1}$, T. Helliwell ${ }^{1}$, D. van Schaardenburg ${ }^{3}$, A. van der Helm-van Mil ${ }^{4}$, K. Raza ${ }^{5}$, C.D. Mallen ${ }^{1} .{ }^{1}$ Arthritis Research UK Primary Care Centre, Keele University, Keele; ${ }^{2}$ Haywood Academic Rheumatology Centre, Haywood Hospital, Staffordshire, United Kingdom; ${ }^{3}$ Reade, Amsterdam; ${ }^{4}$ Department Rheumatology, Leiden University Medical Centre, Leiden, Netherlands: ${ }^{5}$ Rheumatology Research Group, College of Medical and Dental Sciences, University of Birmingham, Birmingham, United Kingdom

Background: Early, accurate diagnosis of RA is critical to improving outcomes. Patients with RA may develop a variety of symptoms including joint pain, swelling and stiffness. The Symptoms in Persons at Risk of Rheumatoid Arthritis (SPARRA) questionnaire was derived to assess the presence, severity and impact of common symptoms in patients at risk of RA (1). However, to date there is little data available on how common these symptoms are in primary care consulters. Objectives: To describe the prevalence of self-reported inflammatory joint symptoms in primary care patients consulting for both musculoskeletal and non-musculoskeletal complaints.

Methods: Questionnaires were sent to 10161 individuals, of whom 5050 had consulted primary care for musculoskeletal problems. The remainder were matched to this sample by age, gender and general practice and had consulted for any non-musculoskeletal indication. Respondents provided data on presence of common symptoms such as joint pain, stiffness and swelling. The prevalence of these symptoms, their severity and impact was compared between musculoskeletal and non-musculoskeletal consulters.

Results: 4549 people responded to the survey (adjusted response $45.8 \%$ ) of whom $52.3 \%$ were in the musculoskeletal consultation group. The mean (SD) age was $61.6(14.8)$ years and $58.9 \%$ were female. Symptoms commonly associated with inflammatory arthritis were common in both groups. $89.1 \%$ of musculoskeletal consulters reported current joint pain, compared with $74.9 \%$ in the non-MSK consulter group, $48.7 \%$ of MSK consulters reported joint swelling compared to $37.3 \%$ of non-MSK consulters and $77.9 \%$ of MSK consulters reported joint stiffness $(64.3 \%$ in non-MSK group). A similar proportion in each group reported fatigue $(59.5 \%$ vs $55 \%)$. Joint symptoms remained common symptoms in both groups even when severity and impact of symptoms was considered.

Conclusions: Although symptoms such as joint pain, swelling, and stiffness are predictive of inflammatory arthritis, a large proportion of those consulting primary care for non-musculoskeletal reasons routinely report these symptoms when prompted. This compounds the challenges of diagnosing inflammatory arthritis in a non-specialist setting where new approaches are needed to ensure accurate, early diagnosis, facilitating a treat to target approach.

References:

[1] van Tuyl, et al. Musculoskeletal Care 2016;14:169-73.

Acknowledgements: This study represents independent research funded by the National Institute for Health Research (NIHR). TH is funded by a NIHR Clinical Lectureship in General Practice. CDM is funded by the National Institute for Health Research (NIHR) Collaborations for Leadership in Applied Health Research and Care West Midlands, the NIHR School for Primary Care Research and a NIHR Research Professorship in General Practice (NIHR-RP-2014-04-026). The views expressed are those of the authors and not necessarily those of the NHS, NIHR or the Department of Health.

Disclosure of Interest: None declared

DOI: 10.1136/annrheumdis-2017-eular.5503

\section{SAT0709 MORTALITY PREDICTION IN MIXED CONNECTIVE TISSUE DISEASE}

S. Reiseter ${ }^{1}$, R. Gunnarsson ${ }^{2}$, T.M. Aaløkken ${ }^{3}$, M.B. Lund ${ }^{4}$, J. Corander ${ }^{5}$, Ø. Molberg ${ }^{6} .{ }^{1}$ Institute of Clinical Medicine, University of Oslo, Norway; ${ }^{2}$ Dept. of Rheumatology; ${ }^{3}$ Dept. of Radiology and Nuclear Medicine; ${ }^{4}$ Dept. of Respiratory Medicine, Oslo University Hospital; ${ }^{5}$ Institute of Basic Medical Sciences;

${ }^{6}$ Institute of Clinical Medicine, University of Oslo, Oslo, Norway

Background: Mixed Connective Tissue Disease (MCTD) is a chronic, immune-mediated disorder defined by the combined presence of serum antiribonucleoprotein (RNP) antibodies and selected clinical features of Systemic Sclerosis, Systemic Lupus Erythematosus, Rheumatoid Arthritis and Polymyositis. Several clinical manifestations and laboratory findings have been found to be associated to increased risk of mortality in univariable analyses (1).

Objectives: Here we present a mortality predicting model in a long-term observational unselected nationwide cohort aiming to enhance the knowledge of long-term prognosis in MCTD.

Methods: 135 patients were included from our nationwide MCTD cohort. Abnormal high resolution computed tomography (CT) findings of ground glass attenuation and reticular patterns were defined as Interstitial Lung Disease (ILD) and expressed as percentage of Total Lung Volume (TLV). Pulmonary function tests and laboratory tests were performed within 2 months of the HRCT examination. Pleuritis was defined as typical pleurisity for more than one day, pleural effusions or pleural rub present at or before baseline. Pericarditis was defined as typical pericardial pain for more than one day, pericardial effusion, pericardial rub or pericarditis by electrocardiography at or before baseline. Myositis was confirmed by muscle biopsy and/or electromyogram and CK elevation at or before baseline. Cox regression analyses were used to find the predictive factors of mortality. Variables at a significant level of $P<.25$ where considered a candidate in the prediction model by manual backward elimination procedure.

Results: 21 patients died after a mean (standard deviation) observation of 9 (2) years. The predictive model is shown in Table 1. According to the Harrell's C index, patient outcomes were accurately predicted by this model $85 \%$ of the time.

Table 1

\begin{tabular}{lcccccc}
\hline & \multicolumn{3}{c}{ Univariable } & & \multicolumn{3}{c}{ Multivariable } \\
\hline & $\mathrm{HR}$ & $95 \% \mathrm{Cl}$ & $\mathrm{P}$ value & $\mathrm{HR}$ & $95 \% \mathrm{Cl}$ & P value \\
Pericarditis ever & 4.0 & $1.6-9.7$ & 0.003 & 5.5 & $1.9-16.0$ & 0.002 \\
Male gender & 2.5 & $1.1-5.9$ & 0.038 & 5.8 & $1.9-17.9$ & 0.002 \\
\% ILD of TLV & 1.1 & $1.03-1.1$ & 0.001 & 1.1 & $1.0-1.1$ & 0.010 \\
DLCO <60\% & 3.1 & $1.3-7.5$ & 0.011 & 3.1 & $1.0-9.2$ & 0.046 \\
Agegroups* at diagnosis & 1.8 & $1.4-2.4$ & $<0.001$ & 1.9 & $1.4-2.5$ & $<0.0001$ \\
Baseline ESR $>30 \mathrm{~mm}$ & 3.3 & $1.4-7.7$ & 0.007 & & & \\
FVC $<75 \%$ & 3.6 & $1.4-8.8$ & 0.006 & & & \\
Arthritis present at or before baseline & 2.1 & $0.85-5.2$ & 0.109 & & & \\
Pleuritis ever & 2.2 & $0.81-6.1$ & 0.121 & & & \\
Baseline Hb $<12 \mathrm{~g} / \mathrm{dL}$ & 2.7 & $1.0-7.0$ & 0.041 & & & \\
Myositis & 0.22 & $0.03-1.7$ & 0.144 & & &
\end{tabular}

*Patients were divided in 6 age groups at diagnosis ( $<25$ years, 26 to 35 years, 36 to 45 years, 46 to 55 years, $56-65$ years and above 65 years). FVC = Forced Vital Capacity \% of predicted, DLCO = diffusing capacity of the lung for carbon monoxide \% of predicted, ESR = erythrocyte sedimentation rate, $\mathrm{TLV}=$ total lung volume, $\mathrm{Hb}=$ Haemoglobin

Conclusions: The strongest predicting factors of mortality in MCTD is increasing $\%$ ILD of TLV, pericarditis, male gender, DLCO less than $60 \%$ of predicted and increasing age at diagnosis.

References:

[1] Hajas A, Szodoray P, Nakken B, Gaal J, Zold E, Laczik R, et al. Clinical course, prognosis, and causes of death in mixed connective tissue disease. The Journal of rheumatology. 2013:40(7):1134-42.

Disclosure of Interest: None declared

DOI: 10.1136/annrheumdis-2017-eular.1855

\section{SAT0710 RENAL FUNCTION CONTRIBUTE TO RISK OF CARDIOVASCULAR DISEASE IN RHEUMATOID ARTHRITIS}

S. Hannawi ${ }^{1}$, I. Alsalmi ${ }^{2}$. ${ }^{1}$ Rheumatology, Ministry of Health and Prevention of UAE, Dubai, United Arab Emirates; ${ }^{2}$ Renal, Royal Hospital, Muscat, Oman

Background: The excess mortality associated with rheumatoid arthritis (RA) is due largely to cardiovascular disease. This highest risk is not related primarily to traditional cardiovasculr/atherosclerosis risk factors. The presence of RA indepedently, as well as high inflammation associated with RA has been reported as a cardiovascular risk factors. Also, subclinical decreased kidney function has been identified as an independent risk factor for $\mathrm{CV}$ events in patients with RA. The potential impact of impaired kidney function on atherosclerosis in RA requires more elucidation.

Objectives: To assess the role of renal parameters, alongside with inflammation and traditional cardiovascular risk factors in predicting cardiovascular disease; as manifested by carotid intima media thickness (cIMT), among RA population.

Methods: cIMT measurement was carried out in 68 RA patients, and correlated with renal function parameters with adjustment for traditional CV risk factors and RA associated inflammation. Glomerular filtration rate (GFR) was estimated with the abbreviated Modification of Diet in Renal Disease formula. Linear regression determined the association between renal parameters and the thickness of cIMT. 
Results: Carotid intima media thickness was positively associated with 1demographic characteristics of the participants such as age of the participants $(p<0.001)$, and age at RA sumptoms onset $(p=0.001)$. 2-traditional cardiovascular risk factors such as systolic blood pressure $(p<0.001)$, diastolic blood pressure $(p=0.016)$, triglycerid level $(p=0.016)$, and low densilty lipoprotein $(L D L)(p=0.001)$. 3 -inflammatory markers such as erythrocytes sedimentation rate (ESR) $(p=0.020)$ and c-reactive protein (CRP) (0.020), and 4-renal function parameters such as uric acid level $(p=0.006)$, urine microalbumin level $(p=0.030)$. cIMT negativlely associated with high density lipoprotein (HDL) $(p=0.037), 24$ hours urine creatinine level $(p=0.020)$ and glomerular filtration rate $(p=0.008)$.

Conclusions: Subclinical renal function in conjunction with tarditional and non-traditional cardiovascular risk factors work synergistically to accelerate atheroscrlerosis in RA population

\section{References:}

[1] Maradit-Kremer H, Crowson, Nicola PJ, Ballman KV, Roger VL, Jacobsen, Gabriel SE. Increased unrecognized coronary heart disease and sudden deaths in rheumatoid arthritis: a population-based cohort study. Arthritis Rheum, 2005:52:402-411.

[2] Roman MJ, Moeller E, Davis A, Paget SA, Crow MK, Lockshin MD, Sammaritano L. Preclinical carotid atherosclerosis in patients with rheumatoid arthritis: prevalence and associated factors. Ann Intern Med, 2006; 144:249256.

[3] van Sijl AM, van den Oever IA, Peters MJ, et al. Subclinical renal dysfunction is independently associated with cardiovascular events in rheumatoid arthritis: the CARRE Study. AnnRheum Dis. 2012;71(3):341-344.

Disclosure of Interest: None declared

DOI: 10.1136/annrheumdis-2017-eular.1664

\section{SAT0711 CHECKPOINT INHIBITOR THERAPY IN PATIENTS WITH ADVANCED MALIGNANCIES AND PREEXISTING RHEUMATOLOGIC DISEASE: THE MAYO CLINIC EXPERIENCE}

U. Thanarajasingam ${ }^{1}$, O. Pinkston ${ }^{2}$, L. Kottschade ${ }^{3}$, H. Finnes ${ }^{3}$, S. Markovic ${ }^{3}$. ${ }^{1}$ Rheumatology, Mayo Clinic, Rochester; ${ }^{2}$ Rheumatology, Mayo Clinic, Jacksonville; ${ }^{3}$ Oncology, Mayo Clinic, Rochester MN, Rochester, United States

Background: Immune checkpoint inhibitors (specifically the anti-CTLA-4 antibody ipilimumab, and the anti-PD-1 antibodies nivolumab and pembrolizumab, have revolutionized the treatment of advanced malignancies. However, by virtue of their mechanism of action - that is, loss of T-cell inhibition and impaired self-tolerance, patients with underlying autoimmune and rheumatologic diseases were typically excluded from the clinical trials leading to the approval of these agents. Limited data, typically of single checkpoint inhibitors, exist for their safety in patients with autoimmune diseases. However, the risk for rheumatologic disease flare in patients exposed to checkpoint inhibitors is unknown.

Objectives: To determine the risk of rheumatologic disease flare in patients receiving checkpoint inhibitor therapy.

Methods: We retrospectively studied all patients who had received a checkpoint inhibitor (i.e. ipilimumab, nivolumab, pembrolizumab, or any combination thereof) for any malignancy on the Mayo Clinic Rochester, Minnesota campus between January 1st, 2011 and May 16th, 2016 (approximately 5,200 patients.) Of these patients, we identified those with preexisting rheumatologic disease according to specific diagnostic codes.

Results: Of the 16 patients identified (13 [81\%] female; median [range] age, 68.5 [34-86]y), 6 had inflammatory arthritis, 3 had polymyalgia rheumatica, 2 had lupus, 2 had Sjogren's syndrome, 1 had temporal arteritis, 1 had IBD-associated spondyloarthropathy and 1 had gout. Patients were treated for the following metastatic/advanced malignancies: melanoma $(9[56 \%])$, lung $(5[31 \%])$ and lymphoma (2 [12\%]) with; ipilimumab (4 [25\%]), nivolumab (7 [44\%]), pembrolizumab $(5[31.2 \%])$ and ipilimumab/nivolumab (1 [6\%]). Notably, in all cases, checkpoint inhibitor therapy was offered after failure of numerous other chemotherapies. Ten (62.5\%) patients were on immunosuppressive therapy (mainly low dose prednisone and methotrexate) at the time of cancer diagnosis, and the majority (10 [62.5\%]) were well-controlled/in remission from the standpoint of their rheumatologic disease. Three (19\%) patients had flares of their rheumatologic disease after treatment for the following cancers as noted: Patient 1 - temporal arteritis nivolumab - non-small cell lung cancer, Patient 2 - Sjogren's (severe sicca)- pembrolizumab - melanoma, and Patient 3 - spondylitis-ipilimumab/pembrolizumab combination -melanoma. All flares responded to steroids or supportive therapy. Checkpoint inhibitor therapy was discontinued in all three patients for the following reasons: Patient 1 - flare of temporal arteritis, Patients $2 \& 3$ - cancer progression. Conclusions: To our knowledge, we have identified the largest cohort (16) of patients from a single academic with preexisting rheumatologic disease who had been exposed to checkpoint inhibitor therapy for advanced malignancy. Of these patients, only a minority experienced a flare of their disease during cancer treatment, and responded to standard therapies. With close monitoring and in the appropriate clinical context, checkpoint inhibitor therapies should be considered and made available to patients with preexisting rheumatologic disease who develop advanced malignancies.

Disclosure of Interest: None declared

DOI: 10.1136/annrheumdis-2017-eular.5461

\section{SAT0712 SELF-REPORTED SEDENTARY BEHAVIOUR IS ADVERSELY ASSOCIATED WITH MICROVASCULAR ENDOTHELIAL FUNCTION IN PATIENTS WITH RHEUMATOID ARTHRITIS}

S.A.M. Fenton ${ }^{1,2}$, J.J. Veldhuijzen van Zanten ${ }^{1,2}$, A. Sandoo ${ }^{3}$, J.L. Duda ${ }^{1}$ G.S. Metsios ${ }^{2,4}$, G.D. Kitas ${ }^{2} .{ }^{1}$ School of Sport Exercise and Rehabilitation Sciences, University of Birmingham, Birmingham; ${ }^{2}$ Rheumatology, Russells Hall Hospital, Dudley; ${ }^{3}$ School of Sport, Health and Exercise Sciences, Bangor University, Gwynedd, Wales; ${ }^{4}$ Faculty of Education Health \& Wellbeing, University of Wolverhampton, Wolverhampton, United Kingdom

Background: Patients with Rheumatoid Arthritis (RA) are at increased risk for cardiovascular disease (CVD). Research suggests impaired vascular function contributes to this heightened risk. At present, little is known regarding factors associated with vascular function in RA. Epidemiological evidence demonstrates sedentary behaviour (i.e., waking behaviour $\leq 1.5$ metabolic equivalents whilst sitting or lying), to be adversely linked to CVD risk in the general population. Whilst the biological processes underlying this relationship are not understood, vascular dysfunction may play a role $(1,2)$. However, research is yet to examine the association between sedentary behaviour and vascular function in healthy adults and/or clinical populations. Studies investigating this relationship in RA, will help to determine the extent to which sedentary behaviour may represent a modifiable risk factor for CVD in these patients.

Objectives: To investigate the cross-sectional associations between sedentary behaviour and microvascular and large vessel endothelial function among patients with RA.

Methods: Fifty-three patients with RA participated in the study $(M$ age $=52.9 \pm 12.8$, $72 \%$ female). Laser Doppler imaging with iontophoresis was used to assess microvascular endothelium-dependent (acetylcholine, ACh) and endotheliumindependent (sodium nitroprusside, SNP) function. Large vessel endotheliumdependent and endothelium-independent functions were measured via flowmediated dilation (FMD) and glyceryl trinitrate dilation (GTN), respectively. Sedentary behaviour was self-reported via the International Physical Activity Questionnaire (hours/week sitting). Data were analysed using multiple linear regressions adjusted for traditional CVD risk factors; age, gender, total cholesterol, smoking status, family history of CVD, hypertension and body-mass-index.

Results: Sitting time (hours/week, $M=39.2 \pm 17.9$ ) was significantly negatively related to $\%$ increase in perfusion in response to ACh $(\beta=-0.30, p<0.05)$ and SNP $(\beta=-0.37, p<0.01)$ after adjustment for traditional CVD risk factors. Sitting time accounted for $8 \%$ and $12 \%$ of the variance in microvascular endothelium-dependent function (ACh) and endothelium-independent function (SNP), respectively (traditional CVD risk factors, $R^{2}=0.3$ ). No significant associations were observed between self-reported sitting time and large vessel endothelium-dependent vasodilation (FMD, $\beta=0.16, p=0.29$ ) or independent vasodilation (GTN, $\beta=-0.08, p=0.55$ ). Conclusions: Sedentary behaviour appears to adversely affect microvascular endothelial function, but not large vessel function in patients with RA. It may therefore represent a modifiable risk factor for CVD in this population. Experimental studies employing objective measures of sedentary behaviour are necessary to confirm these findings, and to determine the utility of sedentary behaviour interventions for improving vascular function and reducing CVD risk in RA

\section{References:}

[1] Thosar SS, Bielko SL, Mather KJ, et al. Effect of Prolonged Sitting and Breaks in Sitting Time on Endothelial Function. Med Sci Sports Exerc 2015:47(4);843-9.

[2] Fenton SAM \& Kitas GD. Rheumatoid Arthritis: Sedentary behaviour in RA a new research agenda. Nat Rev Rheumatol 2016:12(12):698-700.

Disclosure of Interest: None declared

DOI: 10.1136/annrheumdis-2017-eular.4821

\section{SAT0713 SURVEY ON PREVALENCE OF RHEUMATIC DISORDERS IN BANGLADESHI ADULTS}

S. Ahmed ${ }^{1}$, S.A. Haq ${ }^{1}$, A.Z. Al-qadir ${ }^{1}$, M.M. Rahman ${ }^{2}$, S. Paul ${ }^{3}$ ${ }^{1}$ Rheumatology, Bangabandhu Sheikh Mujib Medical University; ${ }^{2}$ Medicine, Dhaka Medical College, Dhaka; ${ }^{3}$ Medicine, Chittagong Medical College, Chittagong, Bangladesh

Background: From Community based COPCORD (community oriented program for control of rheumatic diseases) study in Bangladesh about a quarter of people is suffering from musculoskeletal disorders. But the study was conducted over a small number of samples and some specific localities of the country. So further study was required, covering the whole Bangladesh to justify this high prevalence. Objectives: To determine the prevalence of musculoskeletal symptoms and specific rheumatic disorders in adult population of Bangladesh.

Methods: In this survey, a total of 2000 individuals aged 18 years or older were selected in twenty clusters (primary sample unit) from the seven divisions of the country. Modified COPCORD (Community Oriented Program for Control of Rheumatic Disorders) questionnaire was used to detect positive respondents. Standard criteria were used for diagnosing rheumatic disorders. Clinical judgment was used to solve diagnostic problems.

Results: In total 1843 individuals were interviewed with a response rate of $92.1 \%$. The point prevalence of musculoskeletal pain was $33.7 \%$. It was higher in women $(38.7 \%)$ than men $(28.4 \%)$ and higher in rural $(34.5 \%)$ than that in urban $(32.4 \%)$ areas. Higher prevalence rates were observed in homemakers $(16.0 \%)$, laborers 